\title{
GROUP OF A SET OF SIMULTANEOUS ALGEBRAIC EQUATIONS*
}

BY LOUIS WEISNER

1. Introduction. Consider a system of $m$ independent and consistent algebraic equations in $m$ variables

$$
f_{k}\left(z_{1}, \cdots, z_{m}\right)=0, \quad(k=1, \cdots, m) .
$$

Let the roots of this system of equations be $\left(z_{1 a}, \ldots z_{m a}\right)$ $(a=1, \cdots, n)$. We assume that each $z_{p \alpha}$ is finite. Therefore by a process of elimination we obtain equations

$$
F_{p}\left(z_{p}\right)=0, \quad(p=1, \cdots, m),
$$

each of which is of degree $n$ and involves only one of the unknowns. None of these equations vanishes identically, since equations (1) are independent. We assume that none of equations (2) has a multiple root.

A rational domain $R$ which includes the coefficients of (1) includes the coefficients of (2). It will be shown in this paper that the groups of equations (2) relative to $R$ are identical, except for the symbols which they affect.

2. Elimination of $z_{m}$. On eliminating $z_{m}$ from (1) we obtain $m-1$ equations involving $z_{1}, \cdots, z_{m-1}$. Now $z_{m-1}$ cannot be absent from all of these equations; for in that case we would have $m-1$ equations involving fewer than $m-1$ unknowns, and equations (1) would not be consistent and independent as assumed. Repeating this argument we can find two independent and consistent equations

$$
g_{1}\left(z_{1}, z_{2}\right)=0, \quad g_{2}\left(z_{1}, z_{2}\right)=0,
$$

involving the unknowns $z_{1}, z_{2}$ (or any other pair of the unknowns). This elimination can always be carried out in such a way that if $\left(z_{1 a}, z_{2 a}\right)$ is a root of equations (3), then there is a root $\left(z_{1 a}, z_{2 a}, \cdots, z_{m a}\right)$ of equations (1).

* Presented to the Society, December 27, 1923. 
3. Relations of Rationality. Let $R^{\prime}$ be the domain obtained by adjoining $z_{2 a}$ to $R$. By equations (3), $g_{1}\left(z_{1 a}, z_{2 a}\right)$ and $g_{2}\left(z_{1 a}, z_{2 a}\right)$ are rational functions of a root of $F_{1}\left(z_{1}\right)=0$, with coefficients in $R^{\prime}$ which equal a number (zero) in $R^{\prime}$; they are therefore unaltered in value by the substitutions of the group $H$ of $F_{1}\left(z_{1}\right)=0$ relative to $R^{\prime}$. Suppose that a substitution of $H$ changes $z_{1 a}$ to $z_{1 b}$. Applying this substitution to $g_{1}\left(z_{1 a}, z_{2 a}\right)$ and $g_{2}\left(z_{1 a}, z_{2 a}\right)$ we obtain $g_{1}\left(z_{1 b}, z_{2 a}\right)=0$ and $g_{2}\left(z_{1 b}, z_{2 a}\right)=0$. By $\S 2$, there are two roots $\left(z_{1 a}, z_{2 a}, \cdots, z_{m a}\right)$ and $\left(z_{1 b}, z_{2 a}, \cdots, z_{m b}\right)$ of equations (1) with the same value for $z_{2}$. Therefore $F_{2}\left(z_{2}\right)=0$ has a multiple root, contrary to assumption. It follows that $H$ leaves $z_{1 a}$ fixed. Hence $z_{1 a}$ is in $R^{\prime}$ and is a rational function of $z_{2 a}$ with coefficients in $R$.

Theorem 1. If $\left(z_{1 a}, z_{2 a}, \cdots, z_{m a}\right)$ is a root of equations (1), $z_{p a}$ equals a rational function of $z_{q a}$ with coefficients in $R(p, q=1,2, \cdots, m)^{*}$.

4. Group Relations. Let us regard $G_{p}$, the group of $F_{p}\left(z_{p}\right)=0$ relative to $R$, as a substitution group not on the roots $z_{p 1}, z_{p 2}, \cdots, z_{p n}$, but on their second subscripts. Thus (12) simultaneously interchanges $z_{11}$ and $z_{12}, z_{21}$ and $z_{22}$ etc. With this understanding we state our main result.

Theorem 2. The groups of equations (2) relative to $R$ are identical.

We shall prove $G_{1}$ identical with $G_{2}$. By Theorem 1, $z_{p a}=\varphi_{p a}\left(z_{1 a}\right)$ where $\varphi_{p a}$ is a rational function with coefficients in $R$. Hence by equations (1)

(4) $f_{k}\left[z_{1 a}, \varphi_{2 a}\left(z_{1 a}\right), \cdots, \varphi_{m a}\left(z_{1 a}\right)\right]=0,\left(\begin{array}{l}k=1,2, \cdots, m \\ a=1,2, \cdots, n\end{array}\right)$

* The following is a proof which might be offered of this theorem: Eliminate every power of $z_{1}$ from equations (3) except the first; then $z_{1}$ is expressed as a rational function of $z_{2}$ with coefficients in $R$. The objection to this proof is that in the process of elimination the first power of $z_{1}$ may be incidentally eliminated. It is easy to make up examples where this actually occurs. I believe the proof given above is free from objection. 
Now suppose a substitution of $G_{1}$ replaces $z_{1 a}$ by $z_{1 b}$. Then equations (4) become

$$
f_{k}\left[z_{1 b}, \varphi_{2 a}\left(z_{1 b}\right), \cdots, \varphi_{m a}\left(z_{1 b}\right)\right]=0 .
$$

Therefore $\left[z_{1 b}, \varphi_{2 a}\left(z_{1 b}\right), \cdots, \varphi_{m a}\left(z_{1 b}\right)\right]$ is a root of (1). But $\left[z_{1 b}, \varphi_{2 b}\left(z_{1 b}\right), \cdots, \varphi_{m b}\left(z_{1 b}\right)\right]$ is a root of (1), and (2) has no multiple root. Hence $\varphi_{p a}\left(z_{1 b}\right)=\varphi_{p b}\left(z_{1 b}\right)$. We may therefore omit the second subscript of $\varphi_{p a}$ and write

$$
z_{p a}=\varphi_{p}\left(z_{1 a}\right) .
$$

Now let $\psi\left(z_{21}, \cdots, z_{2 n}\right)$ be a rational function with coefficients in $R$ of the roots of $F_{2}\left(z_{2}\right)=0$ which equals a number in $R$. Then by (6) $\psi\left[\varphi_{2}\left(z_{11}\right), \cdots, \varphi_{2}\left(z_{1 n}\right)\right]$ is a rational function with coefficients in $R$ of the roots of $F_{1}\left(z_{1}\right)=0$ which equals a number in $R$ and hence is unaltered by $G_{1}$. But on applying a substitution of $G$ to $\psi\left[\varphi_{2}\left(z_{11}\right), \cdots, \varphi_{2}\left(z_{1 n}\right)\right]$ we find that, in virtue of $(6), z_{21}, \cdots, z_{2 n}$ undergo a similar substitution. Therefore $\psi\left(z_{21}, \cdots, z_{2 n}\right)$ is unaltered by every substitution of $G_{1}$. It follows that $G_{1}$ is a subgroup of $G_{2}$. Similarly $G_{2}$ is a subgroup of $G_{1}$. Hence $G_{1}$ and $G_{2}$ are identical.

5. A Necessary and Sufficient Condition. We shall call $G=G_{p}(p=1, \cdots, m)$ the group of equations (1) relative to $R$. Let $\psi\left(z_{11}, \cdots, z_{1 n} ; \cdots ; z_{m 1}, \cdots, z_{m n}\right)$ be unaltered in value by all the substitutions of $G$. Then so is $\psi\left[z_{11}, \cdots, z_{1 n}\right.$; $\left.\cdots ; \varphi_{m}\left(z_{11}\right), \cdots, \varphi_{m}\left(z_{1 n}\right)\right]$. But the latter is a rational function with coefficients in $R$ of the roots of $F_{1}\left(z_{1}\right)=0$, which is unaltered by $G$. Hence it equals a number in $R$. Conversely, let $\psi\left(z_{11}, \cdots, z_{1 n} ; \cdots ; z_{m 1}, \cdots, z_{m n}\right)$ equal a number in $R$. Then so does $\psi\left[z_{11}, \cdots, z_{1 n} ; \cdots ; \varphi_{m}\left(z_{11}\right), \cdots, \varphi_{m}\left(z_{1 n}\right)\right]$. Since the latter is unaltered by $G$, so is the former.

Theorem 3. A necessary and sufficient condition that a rational function with coefficients in $R$, of the numbers satisfying a set of independent and consistent algebraic equations with coefficients in $R$, be unaltered in value by the group of the equations relative to $R$, is that it equal a number in $R$.

The University of Rochester 